\title{
Periodic emission of droplets from an oscillating electrified meniscus of a low-viscosity, highly conductive liquid
}

\author{
A. J. Hijano and I. G. Loscertales \\ Andalucía Tech, Escuela Técnica Superior de Ingeniería Industrial, Universidad de Málaga, 29071 Málaga, Spain \\ S. E. Ibáñez and F. J. Higuera \\ Escuela Técnica Superior de Ingenieros Aeronáuticos, Universidad Politécnica de Madrid, 28040 Madrid, Spain
}

(Received 15 October 2014; published 20 January 2015)

\begin{abstract}
The generation of identical droplets of controllable size in the micrometer range is a problem of much interest owing to the numerous technological applications of such droplets. This work reports an investigation of the regime of periodic emission of droplets from an electrified oscillating meniscus of a liquid of low viscosity and high electrical conductivity attached to the end of a capillary tube, which may be used to produce droplets more than ten times smaller than the diameter of the tube. To attain this periodic microdripping regime, termed axial spray mode II by Juraschek and Röllgen [R. Juraschek and F. W. Röllgen, Int. J. Mass Spectrom. 177, 1 (1998)], liquid is continuously supplied through the tube at a given constant flow rate, while a dc voltage is applied between the tube and a nearby counter electrode. The resulting electric field induces a stress at the surface of the liquid that stretches the meniscus until, in certain ranges of voltage and flow rate, it develops a ligament that eventually detaches, forming a single droplet, in a process that repeats itself periodically. While it is being stretched, the ligament develops a conical tip that emits ultrafine droplets, but the total mass emitted is practically contained in the main droplet. In the parametrical domain studied, we find that the process depends on two main dimensionless parameters, the flow rate nondimensionalized with the diameter of the tube and the capillary time, $q$, and the electric Bond number $B_{E}$, which is a nondimensional measure of the square of the applied voltage. The meniscus oscillation frequency made nondimensional with the capillary time, $f$, is of order unity for very small flow rates and tends to decrease as the inverse of the square root of $q$ for larger values of this parameter. The product of the meniscus mean volume times the oscillation frequency is nearly constant. The characteristic length and width of the liquid ligament immediately before its detachment approximately scale as powers of the flow rate and depend only weakly on the applied voltage. The diameter of the main droplets nondimensionalized with the diameter of the tube satisfies $d_{d} \approx(6 / \pi)^{1 / 3}(q / f)^{1 / 3}$, from mass conservation, while the electric charge of these droplets is about $1 / 4$ of the Rayleigh charge. At the minimum flow rate compatible with the periodic regimen, the dimensionless diameter of the droplets is smaller than one-tenth, which presents a way to use electrohydrodynamic atomization to generate droplets of highly conducting liquids in the micron-size range, in marked contrast with the cone-jet electrospray whose typical droplet size is in the nanometric regime for these liquids. In contrast with other microdripping regimes where the mass is emitted upon the periodic formation of a narrow capillary jet, the present regime gives one single droplet per oscillation, except for the almost massless fine aerosol emitted in the form of an electrospray.
\end{abstract}

DOI: 10.1103/PhysRevE.91.013011

PACS number(s): 47.55.-t, 47.65.-d

\section{INTRODUCTION}

Atomization of liquid menisci by means of electric fields has been known for a long time. The evolution of the meniscus interface during this breaking process (and so its outcome) may be quite different depending on the values of the liquid properties, electric field strength, etc., a circumstance that has led to the classification of the electrohydrodynamic (EHD) atomization in different modes. Comprehensive reviews of the main modes have been given by Cloupeau and Prunet-Foch [1] and by Jaworek and Krupa [2]. The first review considers modes appearing in situations where the liquid is continuously fed at a constant rate to a meniscus attached at the tip of a capillary needle. Possibly the most studied mode among these is the so-called steady cone jet, in which a meniscus subjected to an intense electric field adopts a conical shape from whose tip a very thin, highly charged, steady liquid jet is emitted. The breakup of this jet yields a spray of highly charged droplets. The success of this particular mode became apparent when
Fenn et al. [3] discovered that this simple process provides a means to transfer very large ions from the liquid to the gas phase, making them suitable for their posterior weighting in a mass spectrometer. However, the interest in cone-jet electrosprays had already been ignited before, when it was realized that this mode allows generation of monodisperse sprays of droplets whose diameter bears no relation to the diameter of the meniscus. The study of the cone-jet electrospray [4] led to the conclusion that, as the liquid electric conductivity increases, the liquid flow rate has to be decreased and the droplet size decreases as well. Quantitatively, liquids with moderate conductivities (in the order of $10^{-3} \mathrm{~S} / \mathrm{m}$ ) produce monodisperse droplets in the size range of a few microns, whereas liquids with larger conductivities (on the order of $1 \mathrm{~S} / \mathrm{m}$ or above) yield monodisperse droplets in the nanometer range, regardless of the size of the capillary tip. This is a very interesting option to generate ultrafine monodisperse droplets. Therefore, the potential applications of cone jets as 
a monodisperse particle generator have been focused into the submicron and nanometric size ranges [5-7].

There are, however, numerous technological applications in which the droplets, although monodisperse in size, do not need to be so small. A typical example is printing, where the droplet size is in the range of 20-100 $\mu \mathrm{m}$ (see Ref. [8] for a recent review), but there are many others. Combinatorial chemistry, micromixing, or microtritiation [9-11] already focus on monodisperse drop-on-demand systems that yield micron-sized droplets, where the small volumes of the same and the rapid dispensing capabilities make possible an efficient and rapid testing of tens of thousands of mixtures in very short times. Not only that, but performing reactions in airborne microdrops reduces potential contamination from substrates. Analytical tools, such as matrix-assisted laser desorptionionization time-of-flight spectroscopy, also benefit from using microdroplets, not just by saving the use of very expensive compounds but also from the increase in sensitivity brought about when small volumes of samples are used [12,13]. Biotechnology is also an active area in which microdrops play a very important role. Already commercially available systems for sorting cells are based on generation of drops of some $10-20 \mu \mathrm{m}$ in diameter, the size needed to contain a single cell [14]. Bioanalysis based on interactions with tagged DNA fragments also require the use of very small volumes of dissolutions containing the DNA fragments, a very expensive material, something made through generation of microdrops [15-17]. The same requirements apply in drug discovery experiments for medical applications $[9,10]$, without forgetting the direct delivery through lung deposition via monodisperse aerosols of droplets with diameters between 3 and $5 \mu \mathrm{m}$. Other applications include optics [18,19], where the microdrops are used as lenses themselves, manufacturing of complex composite solids, such as directional asymmetric metal-ceramic matrices [20,21], direct soldering [22-25], thin-film coatings, and many more.

Almost all of the technologies operating in industry to produce droplets in this size range resort to hydrodynamic processes where the liquid is forced through a hole with a similar diameter. The forcing stimulus is typically a thermally formed bubble or a piezoelectrically driven moving wall. Electrohydrodynamic atomization might be an alternative, but the modes so far reported in the literature either yield monodisperse droplets that are too small (particularly if the liquid is highly conductive) or produce droplets in the right range of sizes but with a size distribution that is too broad [26-28]. It would be interesting to look for an EHD mode that might fulfill both requirements (size and size distribution), maybe relaxing the requirement of using holes of similar sizes.

Attempts at using electrohydrodynamics for printing have been focused in the so-called microdripping regime, in which the electrified meniscus oscillates and emits certain amounts of mass and charge in each oscillation. The first study, by Juraschek and Röllgen [29], relates to unsteady modes that may appear while trying to set up steady cone jets. For a given liquid flow rate, as the voltage increases they identify two pulsating axial modes, I and II, before reaching the steady cone-jet mode. Briefly, in mode I the meniscus undergoes bursts of fast pulsed emissions (on the order of $\mathrm{KHz}$ ) at rather low frequencies (tens of $\mathrm{Hz}$ ), whereas in mode II the emissions are periodic and proceed at much higher frequencies (tens of $\mathrm{KHz}$ ). More recent investigations of pulsating Taylor cones have been carried out by Marginean et al. [30], Chen et al. [31], and Choi et al. [32], who proposed different scaling laws for the pulsation frequency and the masses delivered, while Marginean et al. [33] introduced a classification of axial modes based on three periodic and stationary regimes interspersed with two chaotic regimes. Kim et al. [34], Kang et al. [35], and Lee et al. [36] achieved improved control of the size and emission frequency of the droplets by using pulsed electric fields and partially classified the new dripping modes that appear in these conditions. Higuera et al. [37] numerically analyzed the pulsating emission from a meniscus of an inviscid liquid of infinite electrical conductivity in a simplified configuration, qualitatively reproducing experimental results for constant and pulsed voltages. Further steps toward the practical implementation of these techniques for high-speed and drop-on-demand EHD printing have been taken by Mishra et al. [38] and Sutanto et al. [39].

Many of the works mentioned here are based on the periodic emission of a single liquid jet from the electrified meniscus. This jet is unstable under varicose disturbances and breaks into drops whose diameter is similar to that of the jet. This type of microdripping is useful for direct printing, that is, for applications in which the flowing jet lands on a spot on the target before breaking into droplets, thus forming a single drop of a given volume. However, if the objective is to form drops that may be airborne for a later use, such as in material forming processes, this type of microdripping is seldom useful. A recent use of electrohydrodynamics to generate nanodroplets for printing of nanostructures is that of Galliker et al. [40], where micron-sized nozzles were used to eject monodisperse droplets, with diameters between 100 and $80 \mathrm{~nm}$, of a colloidal suspension of gold nanoparticles. At landing on a substrate, the volatile solvent evaporated thus leaving a dense residue formed by a compact agglomeration of the gold nanoparticles. As nanodroplets were continuously landing and evaporating on the same spot tiny nanopillars, made of agglomerated gold nanoparticles, could be formed. Although these authors could not follow the droplet formation dynamics, their experiments undoubtedly demonstrate that the periodic EHD microdripping can be used to generated monodisperse nanosized droplets from nozzles some 10-15 times larger in diameter. Finally, even though a drawback of EHD techniques is the required electrical conductivity of the liquids, this constraint may also be overcome by special nozzle designs, such as that described by Lee et al. [41], or by the combined use of conductive liquids, such as that used by Larriba and Fernández de la Mora [6], thus broadening the potential classes of liquids that may be used.

Motivated by the potential applications mentioned above, the present work aims at studying an EHD microdripping regime in which most of the mass emitted per pulsation is in the form of a single drop instead of a jet, whose size may be tailored to be much smaller than the capillary tip anchoring the oscillating meniscus and whose droplet emission frequency is comparable to industrially used droplet generation processes. In particular, we shall focus on highly conducting, low-viscosity liquids. 


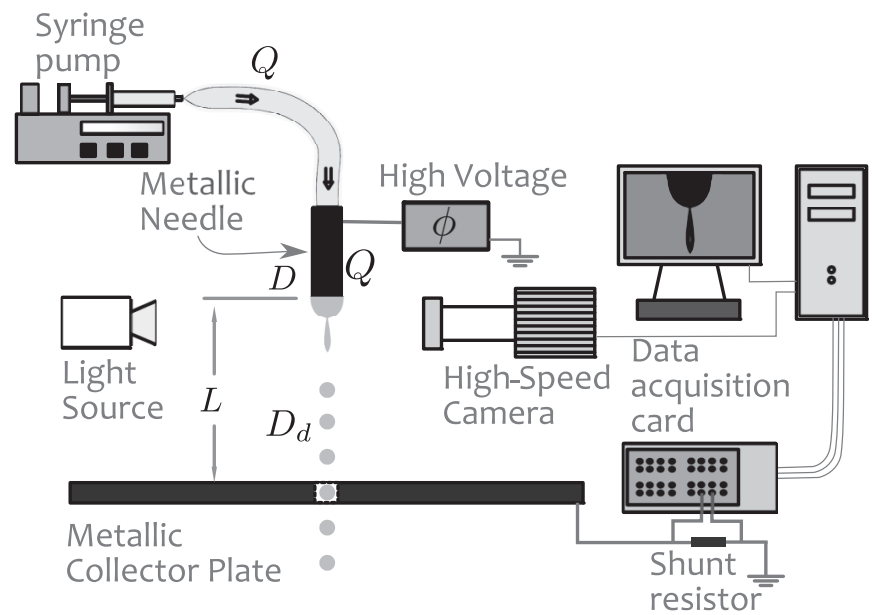

FIG. 1. Scheme of the experimental setup.

\section{EXPERIMENT}

\section{A. Setup and measurement techniques}

The microdripping of the liquid has been studied in the experimental setup sketched in Fig. 1. A long capillary needle of stainless steel (outer diameter $D$ ) is placed vertically at a certain distance $L$ in front of a circular metallic plate. Unless otherwise noted, the dimensions of the needle used are outer diameter $D=500 \mu \mathrm{m}$, inner diameter $200 \mu \mathrm{m}$, and length $20 \mathrm{~mm}$. The needle-to-collector distance is $L=9 \mathrm{~mm}$ and the radius of the collector is $31.5 \mathrm{~mm}$. The liquid to be atomized is supplied at a known flow rate $Q$ by a syringe pump (KDS-100-CE, Kd Scientific). A constant voltage $\phi$ is applied between the needle and the plate by means of a high-voltage power supply (205B-10R, Bertan). The time evolution of the meniscus sticking out of the capillary needle is recorded by a high-speed camera (Fastcam APX-RS, Photron) with an optical system with magnification from 2.3 up to $12.5 \mu \mathrm{m} /$ pixel and the image is monitored on a television screen $(701 \mathrm{~N}$, Samsung). The electric current at the collector plate is measured using a data acquisition card (6024-E, National Instruments). The meniscus is illuminated by using a fiber optic illuminator (4000-1,Volpi) with a 4-mm flexible fiber optic light guide.

The liquid used is a mixture of $64 \%$ water, $11 \%$ ethanol, and $25 \%$ ethylene glycol, with $1.7 \mathrm{mg} / \mathrm{g}$ hydrochloric acid. Unless otherwise stated, the properties of the liquid, measured at $25^{\circ} \mathrm{C}$, are as follows. The surface tension is $\gamma=41.35 \pm$ $0.42 \mathrm{mN} / \mathrm{m}$, measured with a ring tensiometer (Sigma702, $\mathrm{KSV})$. The electric conductivity is $K=4.13 \pm 0.66 \mathrm{mS} / \mathrm{cm}$, measured with a conductimeter (TB84, ABB). We have not measured the dielectric constant of the mixture $\epsilon$; the mean value of the dielectric constants of their components has been arbitrarily taken as the real value. The viscosity is $\mu=$ $2.28 \pm 0.17 \mathrm{mPa}$ s, measured with a custom-made Ostwald viscometer. The density is $\rho=1065 \mathrm{Kg} / \mathrm{m}^{3}$, measured with a pycnometer.

Minimizing the liquid spreading over the needle tip is essential to the robustness and reproducibility of the experiments. A thin layer of an antiwetting agent (602A-FP and 602A-FS, Cytonix) was deposited on the outer part of the needle end (a)

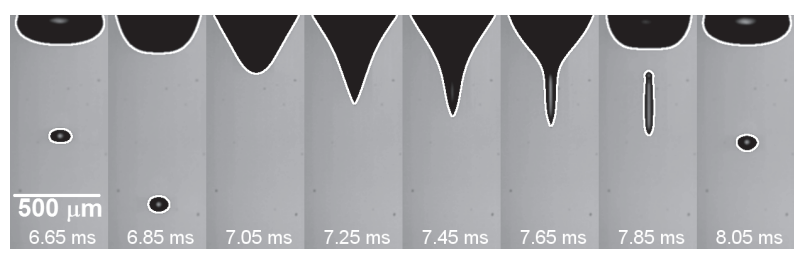

(b)

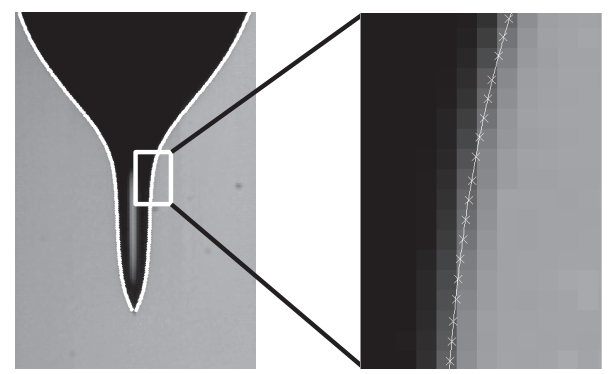

FIG. 2. Interface tracking for periodic microdripping emission with $Q=2.1 \mathrm{ml} / \mathrm{h}, \phi=3.39 \mathrm{kV}, L=9 \mathrm{~mm}$, and $D=500 \mu \mathrm{m}$. (a) Collage of different frames, with recognition of different meniscus and droplet shapes. The shapes that are recognized are marked with a white border. (b) Edge detection. The crosses correspond to the edge detection made by the Canny method after the subpixel correction. The white curve is the fitting of cubic splines to the position of the edges detected.

to ensure that the oscillating meniscus is anchored at the needle rim. The coating process consisted of dipping the needle end in the commercial antiwetting solution and on thermally stabilizing the agent for $60 \mathrm{~min}$ in air at $150{ }^{\circ} \mathrm{C}$.

The volume of the detached droplets has been measured as follows. For a given microdripping situation, the oscillating meniscus and the emitted droplets have been continuously recorded using a high-speed camera, so the time evolution of the meniscus and an emitted droplet as it moves away from the meniscus are obtained; see Fig. 2 for a few sample frames. Each frame of the emitted droplet, which is not spherical due to its own oscillations, has been processed with a custom-made software of interface tracking, which provides the droplet contour in the visualization plane, say $r=r_{d}(z)$, where, ideally, $z$ and $r$ are distances along and normal to the axis of the needle. White curves in Fig. 2(a) show sample detected contours of the meniscus and flying droplets. The volume of the droplet is calculated from its contour under the assumption of axisymmetry. The mean volume of the droplet $V_{\text {mean }}$ and its variance are calculated by processing a large number of frames and the mean volume is accepted if the variance is sufficiently small. The droplet equivalent diameter is then defined as $D_{d}=\left(6 V_{\text {mean }} / \pi\right)^{1 / 3}$.

A similar approach has been used to measure the instantaneous meniscus volume. More precisely, the Canny algorithm [42] has been used to detect the edges of droplets and meniscus in each frame. To increase precision, a subpixel detection method has also been implemented, which consists of fitting a sigmoid function to the gray intensity level across the edge in each point, as in Refs. [43,44]. An example of how the algorithm works is shown in Fig. 2(b). The picture on the left-hand side shows a frame of the oscillating meniscus, where the ligament that eventually will form a droplet can be identified. The picture on the right shows a detail of the 
(a)
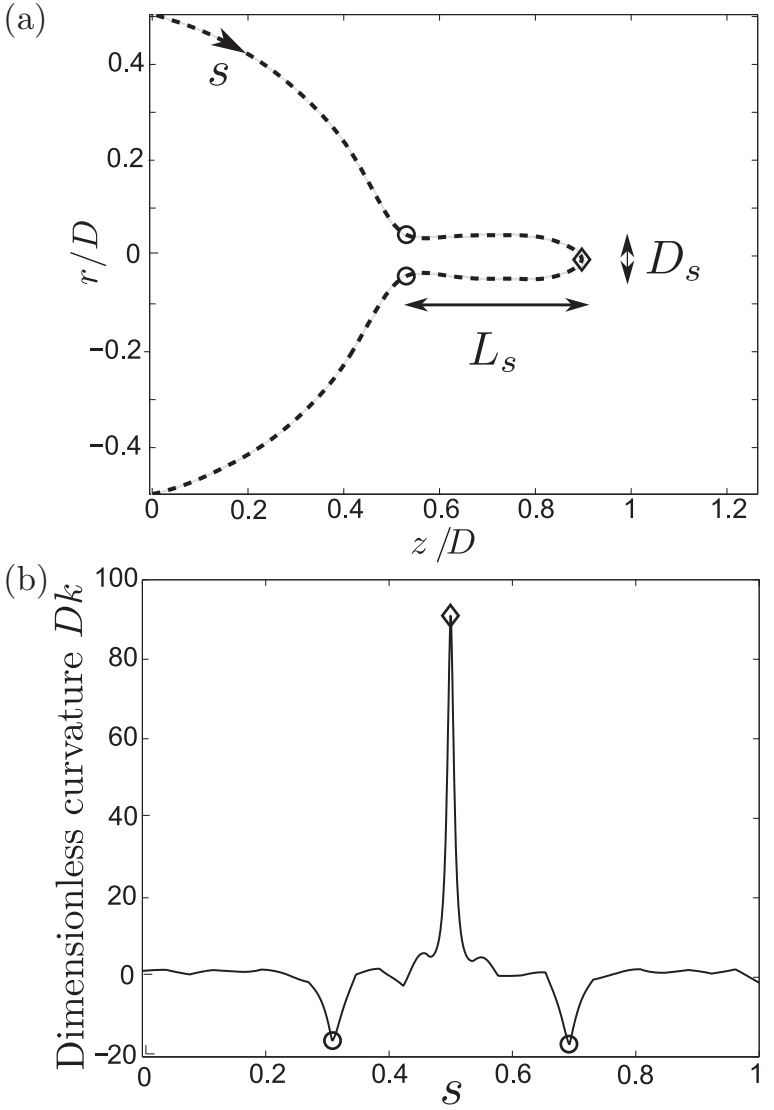

FIG. 3. Detection of the ligament dimensions. (a) Meniscus profile in dimensionless coordinates $r_{m} / D$ and $z_{m} / D$. The dashed line is the least-squares spline interpolation to the detected points of the meniscus from the images, using the subpixel algorithm. The circles and the diamond represent the position of the base and the tip of the ligament, respectively. (b) Dimensionless meniscus curvature $D k$ as a function of arc length $s$ calculated from splines fitted to the meniscus shape. The diamond represents the tip and the circles represent the base of the ligament.

neck forming at its base. The crosses in this picture mark the edge defined by sequentially applying the subpixel and Canny algorithms. The white curve corresponds to the least-squares spline interpolation of the points defining the edge, using different cubic splines. Finally, the spline fitting is used to compute the instantaneous volume of the droplet and the meniscus. The relative variation of the meniscus volume during a cycle of the oscillation is always small when the flow rate is small and increases when this parameter increases.

To measure the length $L_{s}$ and the width $D_{s}$ of the ligament at the tip of the meniscus (see Fig. 3), we measure the local mean curvature of the meniscus surface using the splines fitted to its contour, say $z_{m}(s)$ and $r_{m}(s)$, where $s$ is the arc length on the meniscus contour. The curvature of the meniscus, assumed axisymmetric, is

$$
\begin{aligned}
k(s)= & \frac{z_{m}^{\prime \prime}(s) r_{m}^{\prime}(s)-z_{m}^{\prime}(s) r_{m}^{\prime \prime}(s)}{\left[z_{m}^{\prime}(s)^{2}+r_{m}^{\prime}(s)^{2}\right]^{3 / 2}} \\
& -\frac{z_{m}^{\prime}(s)}{r_{m}(s)\left[z_{m}^{\prime}(s)^{2}+r_{m}^{\prime}(s)^{2}\right]^{1 / 2}},
\end{aligned}
$$

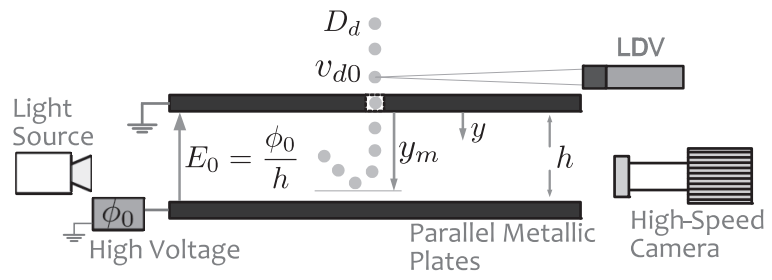

FIG. 4. Setup used to measure the droplets' electric charge.

which is shown in Fig. 3(b) for a sample case. The base (circles) and tip (diamond) of the ligament are then readily located. The length $L_{s}$ of the ligament is defined as the axial distance between these points, while its width $D_{s}$ is the maximum diameter of the ligament cross section, when the arc length $s$ varies between the values corresponding to the base and the tip.

The electric charge carried by the droplets has been measured using the device sketched in Fig. 4. The droplets emitted by the meniscus enter the space between two parallel plates separated a distance $h$ through a small orifice drilled in the upper plate. This plate is grounded, as it is the collector electrode in Fig. 1. The lower plate is set to a voltage $\phi_{0}$, which induces a uniform field $E_{0}=\phi_{0} / h$ between the plates that pushes the droplets toward the upper plate. The velocity $v_{d 0}$ with which the droplets enter the orifice is measured with a laser Doppler velocimeter (miniLDV-G5-240, Measurement Science Enterprise Inc.) and the trajectory of a droplet between the plates is recorded with a high-speed camera. The charge of the droplet $\mathcal{C}_{d}$ is determined by comparing the recorded trajectory with the trajectory computed by solving the problem

$$
m_{d} v_{d} \frac{d v_{d}}{d y}=-\mathcal{C}_{d} E_{0}-\frac{\pi}{8} c_{D} \rho_{a} D_{d} v_{d}\left|v_{d}\right|,
$$

with $v_{d}=v_{d 0}$ at $y=0$, where $m_{d}$ and $v_{d}$ are the mass and velocity of the droplet, $\rho_{a}$ is the density of the air between the plates, and $c_{D}$ is the drag coefficient, given by $c_{D}=(24 / \mathrm{Re})\left(1+0.15 \mathrm{Re}^{0.687}\right)$ in terms of the Reynolds number $\operatorname{Re}=\rho_{a}\left|v_{d}\right| D_{d} / \mu_{a}$, with $\mu_{a}$ the viscosity of the air [45]. The charge $\mathcal{C}_{d}$ is determined from the condition that the lowest point of the droplet's trajectory computed from (2) coincides with that of the recorded trajectory. In many cases, the drag force turns out to be small compared to the electric force in (2) and then the lowest point of the trajectory is $y_{m} \approx m_{d} v_{d 0}^{2} /\left(2 \mathcal{C}_{d} E_{0}\right)$.

\section{B. Oscillation regimes}

The experiments were performed as follows. For a given setup geometry (needle size, needle-to-collector distance, collector geometry, etc.) a flow rate is selected and the voltage is sequentially increased. For zero voltage, the quasispherical meniscus grows until a single drop is detached by the effect of gravity. The diameter of this drop is larger than the diameter of the needle. As the voltage increases, the meniscus gets stretched along the needle axis, the detachment frequency increases, and the size of the detached droplets decreases, although it is still of the order of the needle diameter. For a sufficiently large voltage, a periodic microdripping regime is suddenly attained, such as the one shown in Fig. 5. This regime is identified by a jump in the meniscus oscillation 
(a)

(b)

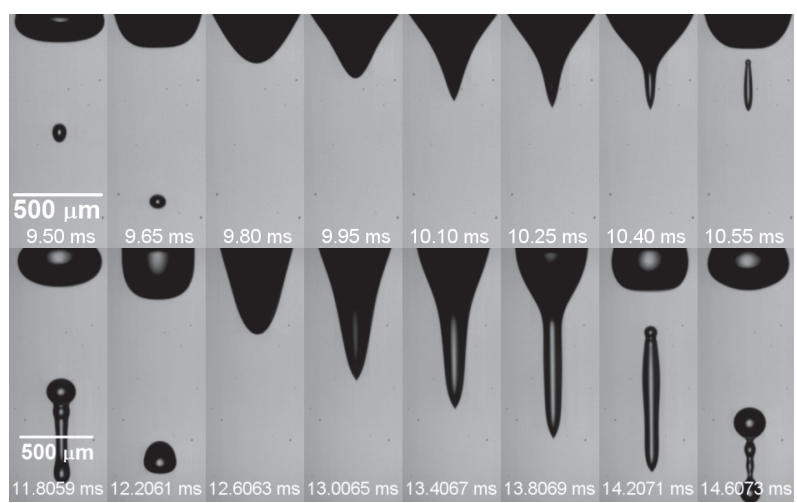

FIG. 5. Snapshots of the microdripping regime for (a) $Q=$ $1.0 \mathrm{ml} / \mathrm{h}$ and (b) $Q=10 \mathrm{ml} / \mathrm{h}$. The values of other parameters are $\phi=3.39 \mathrm{kV}, L=9 \mathrm{~mm}$, and $D=500 \mu \mathrm{m}$.

frequency, by the appearance of a sharp conical tip on the stretched oscillating meniscus, and by a significant reduction of the diameter of the emitted droplets. The motion is axisymmetric. The microdripping regime may be sustained within a certain voltage range. The same protocol is repeated for different flow rates. The voltage upper limit of this periodic microdripping is indicated by either the appearance of nonaxisymmetric motion of the oscillating meniscus, when the flow rate is moderate, or the emission of more than one droplet per oscillation, when it is larger.

Series of experiments have also been carried out in which the flow rate is increased at constant voltage, in order to characterize the range of flow rates compatible with the microdripping regime. For very low flow rates, not all the meniscus oscillations yield a droplet. As we shall see, the minimum flow rate for which periodic microdripping occurs also yields the minimum droplet diameter. The diameter of the emitted droplets increases with the flow rate until eventually the droplets cease to be small compared to the diameter of the capillary tube.

We have recorded the electric current induced at the collector electrode by the mass and charge emission from the oscillating meniscus, using a data acquisition card. The recorded current signal changes as the voltage applied to the needle increases, as shown in Fig. 6. Figure 6(a) shows a highfrequency $(\mathrm{KHz})$ charge emission that occurs intermittently at a much smaller frequency (tens of $\mathrm{Hz}$ ), an electric nonperiodic microdripping termed axial spray mode I by Juraschek and Röllgen [29]. The oscillation of the meniscus becomes periodic for a larger voltage, giving an electric signal such as the one shown in Fig. 6(b). This periodic microdripping is termed axial spray mode II by Juraschek and Röllgen [29]. This behavior, in which the emission regime from the meniscus goes from electrodripping to nonperiodic microdripping (axial spray mode I) and then to periodic microdripping (axial spray mode II) as the voltage increases, only appears in our experiments when the liquid flow rate is small (the lowest value used in the experiments for each voltage). Otherwise, a direct transition from electrodripping to periodic microdripping (axial spray mode II) occurs.
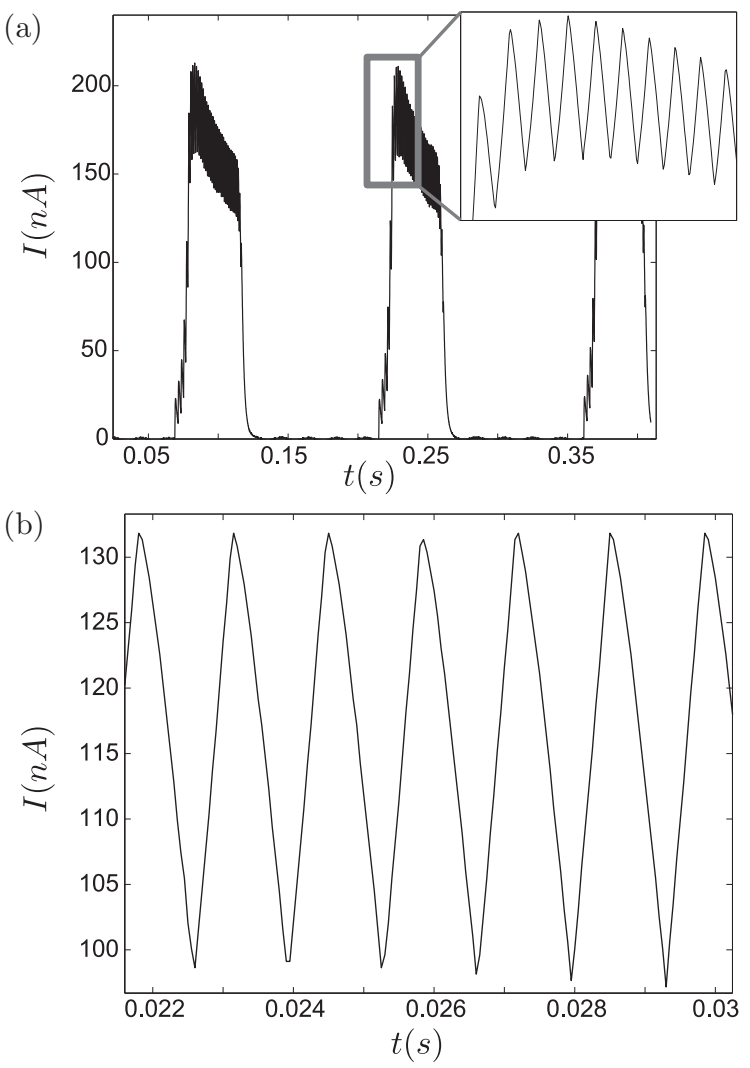

FIG. 6. Current recorded at the collector electrode: (a) nonperiodic microdripping emission (axial spray mode I) $(Q=0.2 \mathrm{ml} / \mathrm{h}$, $\phi=3.45 \mathrm{kV}, L=9 \mathrm{~mm}$, and $D=500 \mu \mathrm{m}$ ) and (b) periodic microdripping emission (axial spray mode II) $(Q=0.5 \mathrm{ml} / \mathrm{h}, \phi=$ $2.95 \mathrm{kV}, L=9 \mathrm{~mm}$, and $D=500 \mu \mathrm{m})$.

\section{Dimensionless variables and results}

The meniscus mean volume $V_{M}$ and oscillation frequency $F$, and the electric charge $\mathcal{C}_{d}$ and equivalent diameter $D_{d}$ of the detached droplets, have been measured for many values of the applied voltage and the flow rate. We shall use dimensional considerations and order-of-magnitude estimations to help organize the large body of data thus gathered. In what follows, distances are scaled with the outer diameter of the needle $D$, where the meniscus is attached, and times are scaled with the capillary time $t_{c}=\left(\rho D^{3} / \gamma\right)^{1 / 2}$. The dimensionless flow rate is

$$
q=\frac{Q}{D^{3} / t_{c}}=\frac{\rho^{1 / 2} Q}{\gamma^{1 / 2} D^{3 / 2}}
$$

and the square of the voltage applied between the needle and collector is measured by the electric Bond number

$$
B_{E}=\frac{\epsilon_{0} E^{2} D}{\gamma} \quad \text { with } \quad E=\frac{\phi}{D \ln (4 L / D)} .
$$

Here $\epsilon_{0}$ is the permittivity of vacuum, $L$ is the needle-to-collector distance, and $E$ is the characteristic electric field induced by the applied voltage around the end of the needle $[32,46]$.

The needle-to-collector distance is large compared to the diameter of the needle; the ratio $L / D$ is about 18 in our experiments. The length of the needle and the radius of the 


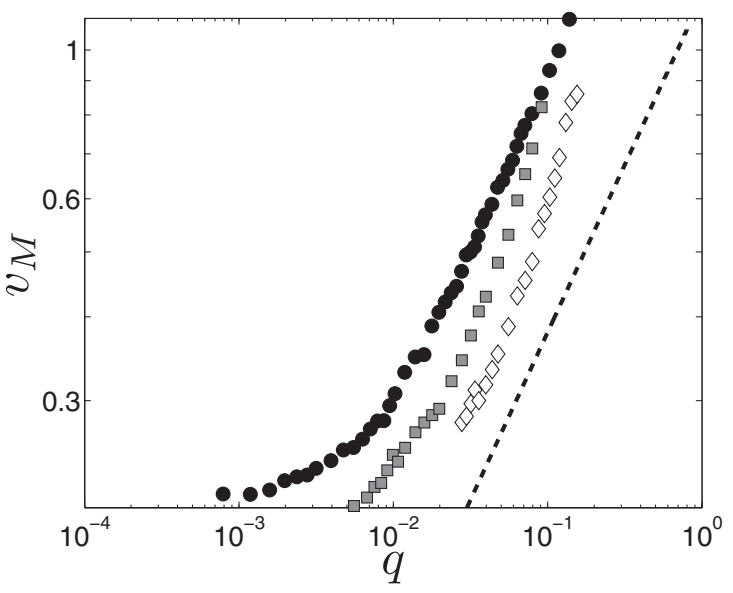

FIG. 7. Mean dimensionless volume of the meniscus $v_{M}=$ $V_{M} / D^{3}$ as a function of the dimensionless flow rate for $B_{E}=0.20$ (circles), 0.27 (squares), and 0.37 (diamonds). The dashed line has slope $1 / 2$.

collector are even larger. In these conditions, the precise values of these two parameters are irrelevant, while $L$ has only a weak effect that is accounted for using the expression of $E$ in (4) above instead of the simpler expression $\phi / D$.

The effect of the viscosity of the liquid $\mu$ is small in our experiments. Taking the capillary velocity $v_{c}=D / t_{c}$ as a characteristic liquid velocity, this effect is measured by the Ohnesorge number $\mathrm{Oh}=\mu /\left(\rho v_{c} D\right)=\mu /(\rho \gamma D)^{1 / 2}$, which is small, of order $10^{-2}$.

The effects of the electrical conductivity $K$ and the dielectric constant of the liquid $\epsilon$ are also small because the electric relaxation time $t_{e}=\epsilon_{0} \epsilon / K$ is small compared to the capillary time, the ratio $t_{e} / t_{c}$ being of order $10^{-7}$. The electric relaxation time is the characteristic time required for the electric charge to reach the surface of the liquid by conduction and screen the liquid from the surrounding electric

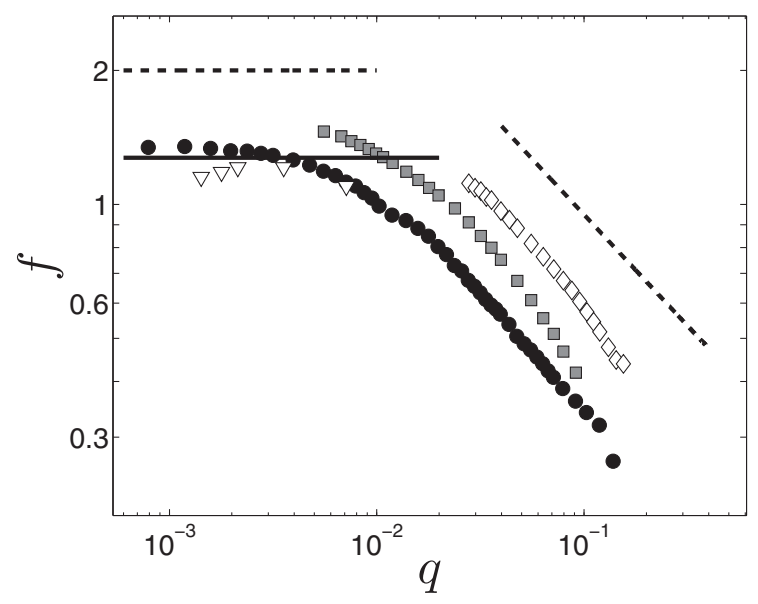

FIG. 8. Dimensionless oscillation frequency $f=F t_{c}$ as a function of the dimensionless flow rate for $B_{E}=0.20$ (circles), 0.27 (squares), and 0.37 (diamonds). The dashed line at the right-hand side has slope $-1 / 2$. Open triangles show data from Fig. 13 of Juraschek and Röllgen [29] for $B_{E}=0.17$. The solid line stands for the expression $f=4 / \pi$ proposed by Marginean et al. [30].

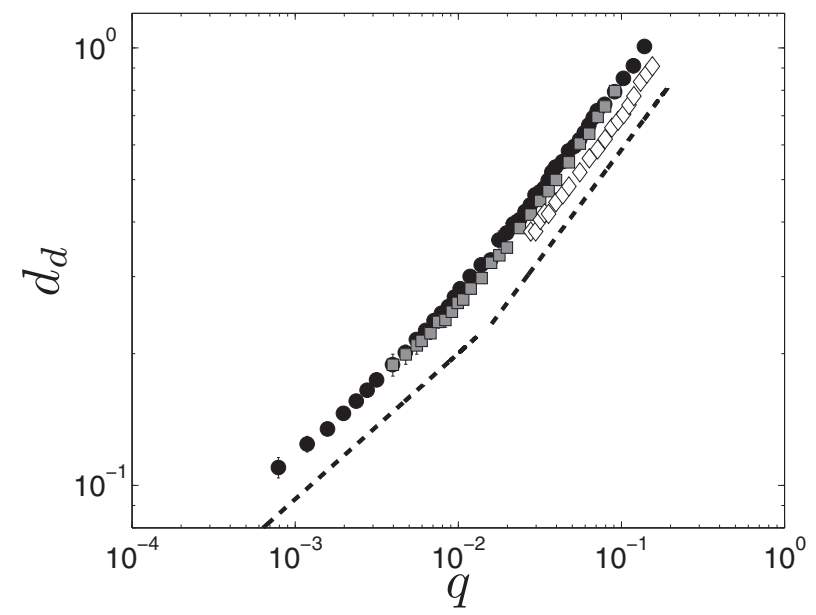

FIG. 9. Dimensionless diameter of the droplet $d_{d}=D_{d} / D$ as a function of the dimensionless flow rate for $B_{E}=0.20$ (circles), 0.27 (triangles), and 0.37 (squares). The dashed line at the left-hand side has slope $1 / 3$ and the dashed line at the right-hand side has slope $1 / 2$.

field; see, e.g., Ref. [47]. Its small value implies that the surface of the liquid is essentially at the potential of the needle in our experiments.

The effect of the gravity acceleration is also small because the Bond number $\rho g D^{2} / \gamma$ is small, of order $10^{-2}$.

In these conditions, the only relevant control parameters are expected to be $q$ and $B_{E}$. Figures 7-9 show the dimensionless volume of the meniscus averaged over a cycle of the oscillation $v_{M}=V_{M} / D^{3}$, the dimensionless frequency of the oscillation $f=F t_{c}$, and the dimensionless equivalent diameter of the droplets $d_{d}=D_{d} / D$ as functions of the dimensionless flow rate $q$ for $B_{E}=0.20,0.27$, and 0.37 .

Figure 10 shows the electric charge of the droplet scaled with the limit Rayleigh charge at which a spherical droplet of diameter $D_{d}$ becomes unstable, $\mathcal{C}_{d} / \mathcal{C}_{R}$ with $\mathcal{C}_{R}=$ $\left(8 \pi^{2} \epsilon_{0} \gamma D_{d}^{3}\right)^{1 / 2}$ (see Ref. [48]) as a function of the electric Bond number $B_{E}$ for three values of $q$. Figure 11 shows the

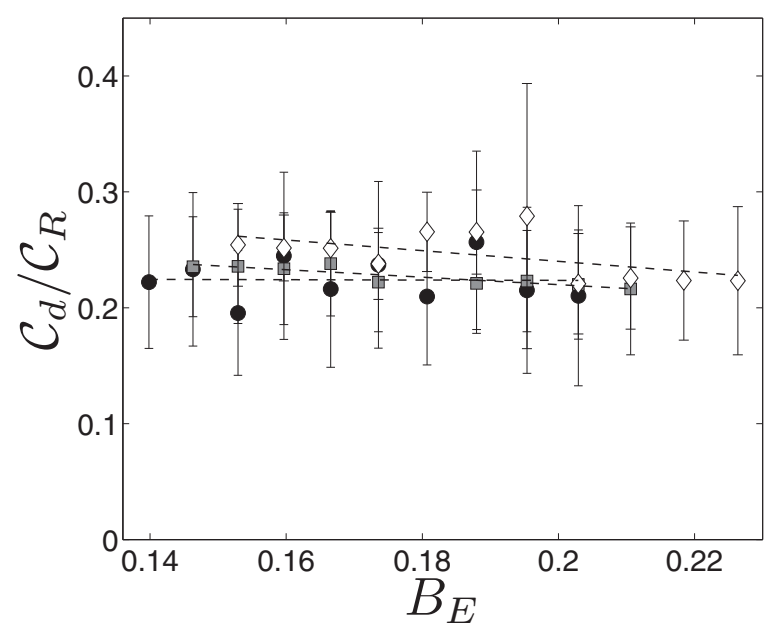

FIG. 10. Dimensionless electric charge of the droplets $\mathcal{C}_{d} / \mathcal{C}_{R}$ as a function of $B_{E}$ for $q=1.6 \times 10^{-3}$ (circles), $4.1 \times 10^{-3}$ (squares), and $1.2 \times 10^{-2}$ (diamonds). 


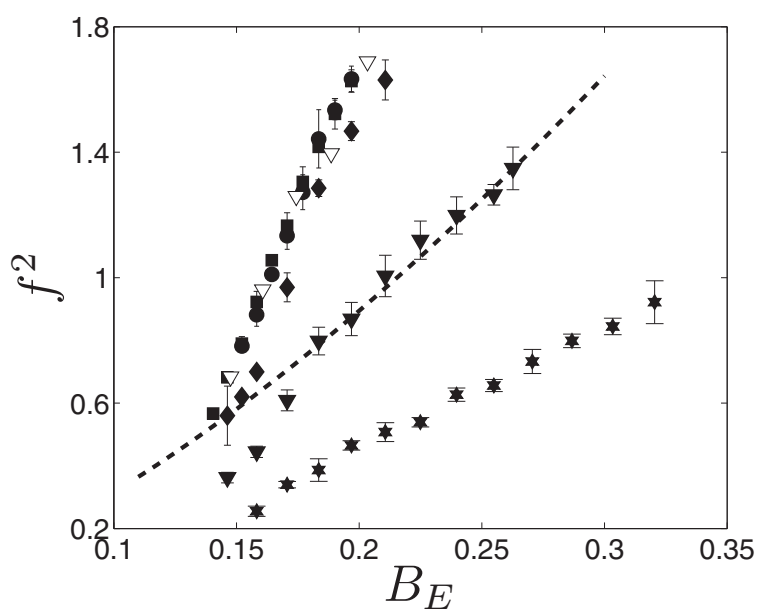

FIG. 11. Square of the dimensionless oscillation frequency as a function of the electric Bond number for $q=8 \times 10^{-4}, 2 \times 10^{-3}$, $4 \times 10^{-3}, 1.47 \times 10^{-2}$, and $3.4 \times 10^{-2}$, increasing from top to bottom (closed symbols). Open triangles show data from Fig. 6 of Juraschek and Röllgen [29] for $q=1.42 \times 10^{-3}$. The dashed line stands for the expression $f \sim B_{E}^{3 / 4}$ proposed by Choi et al. [32].

square of the dimensionless frequency as a function of $B_{E}$ for various values of $q$. All these results are for a needle of outer diameter $D=500 \mu \mathrm{m}$. Dimensionless data collected with capillary needles having different outer diameters also approximately collapse onto the same curves, although these results have not been shown to avoid cluttering the figures.

\section{DISCUSSION}

\section{A. Dimensionless frequency and mean volume}

The microdripping regime analyzed here occurs only for values of the electric Bond number of order unity, in a range that increases somewhat when the flow rate increases; see Fig. 11. Surface tension stresses, of $O(\gamma / D)$, and electric stresses, of $O\left(\epsilon_{0} E^{2}\right)$, are thus of the same order and equally important in the evolution of the meniscus. The electric stress tends to elongate the meniscus axially and the maximum elongation increases with the dimensionless flow rate. Inspection of sequences of images similar to those of Fig. 5 shows that (i) the diameter of the meniscus cross section is not larger than the diameter of the needle $D$ during most of the evolution, while its length, say $H$, may be of the order of $D$ (at small flow rates) or somewhat larger than $D$ (when the flow rate increases) and (ii) the amplitude of the oscillation of the tip is of the order of $H$. An order-of-magnitude balance of the liquid acceleration and the pressure force due to the electrically induced depression reads therefore $\rho H F^{2} \sim \epsilon_{0} E^{2} / H$, whence, in dimensionless variables, $f \sim B_{E}^{1 / 2} / v_{M}$.

In agreement with this estimation, the product $f v_{M}$ computed using data from Figs. 7 and 8 is nearly constant, independent of the flow rate in the whole range of this parameter spanned by our experiments. This product is also nearly independent of $B_{E}$, which may be due to the narrow range of $B_{E}$ where the microdripping regime is realized. With good approximation our results give $f v_{M} \approx 0.32$.

\section{B. Scale disparity: Small $\boldsymbol{q}$ and domain of existence}

The volume of liquid emitted per period of the oscillation is equal to the volume supplied by the imposed flow rate during a period. At small flow rates, this volume is small compared to the volume of the meniscus. The ratio of droplet-to-meniscus volume is then of order $q$ because the period of the oscillation is of the order of the capillary time $[f=O(1)$ on the left-hand side of Fig. 8] and the volume of the meniscus is of the order of $D^{3}\left[v_{M}=O(1)\right.$ on the left-hand side of Fig. 7]. The periodic microdripping at small $q$ entails thus a delicate balance to keep the mean meniscus volume close to the boundary between oscillations without volume loss and more violent oscillations in which electric stresses would tear the meniscus and cause the loss of a significant fraction of its volume.

This critical volume must coincide with the plateau value in Fig. 7, a value that depends on the electrical Bond number. Similarly, the oscillation frequency of a meniscus with this critical volume must coincide with the plateau value in Fig. 8 . This frequency is affected by the presence of the electric field and is different from the capillary frequency of a meniscus of the same volume (but see Ref. [30]). The electric field and the electric stress around the tip of the meniscus intensify when the meniscus elongates, which in turn reinforces the elongation until a conical tip develops. When the meniscus begins to recede, the conical tip lags behind the rest of the meniscus and at the same time shortens and sharpens under the squeezing effect of the surface tension; see Fig. 5(a).

Since the volume of the meniscus is nearly constant when $q$ is small, the problem of finding $v_{M}, f$, and $d_{d}$ as functions of $q$ and $B_{E}$ can be approximately recast as that of finding $f\left(v_{M}, B_{E}\right)$ and $d_{d}\left(v_{M}, B_{E}\right)$ for a meniscus of strictly constant dimensionless volume $v_{M}$ and then making $q=(\pi / 6) d_{d}^{3} f$. Since $\left(v_{M}, B_{E}\right)=O(1)$, this problem should have a solution with $d_{d} \ll 1$ only in a narrow band around the curve of the $\left(v_{M}, B_{E}\right)$ plane where $d_{d}=0$. On one side of this curve the meniscus would oscillate without ejecting any droplet. On the other side of the curve the volume of the droplets, hence $q$, would increase with distance to the curve. To get an idea of the shape of the band and the limiting curve where $d_{d}=0$, Fig. 12

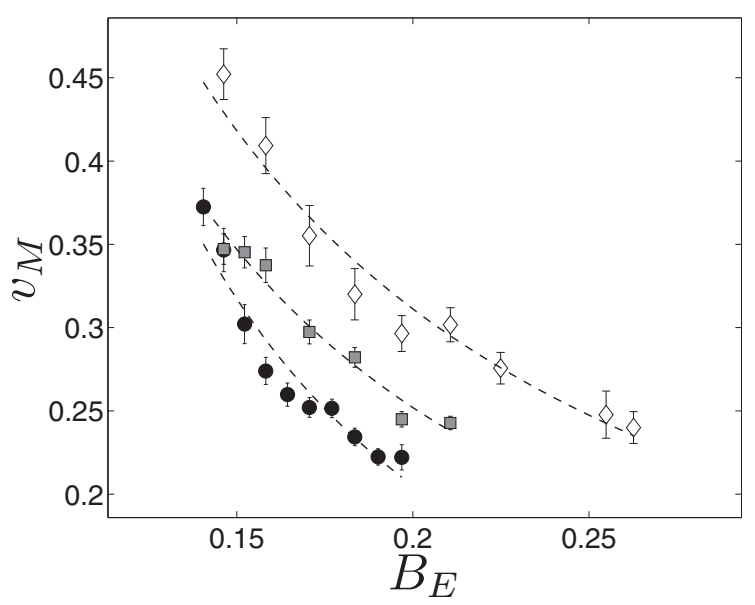

FIG. 12. Contours of constant $q$ in the $\left(v_{M}, B_{E}\right)$ plane. Contours shown are $1.93 \times 10^{-3}$ (circles), $3.86 \times 10^{-3}$ (squares), and $1.43 \times$ $10^{-2}$ (diamonds), increasing from bottom to top. 
shows some contours of constant, small $q$ in the $\left(v_{M}, B_{E}\right)$ plane. The lowest contour should be close to the limiting curve. As can be seen, the range of $B_{E}$ where microdripping occurs extends to larger values of this parameter when $q$ increases.

\section{Tip emission of electrospray}

One of the characteristics of the microdripping regime studied here is the existence of a sharp conical tip that persists during a non-negligible part of the meniscus oscillation cycle, as shown in Fig. 5. This conical tip leads to the formation of an electrospray. Even though we have not attempted to measure or quantify such emission, its existence is confirmed by adding colorant to some of the liquids: A fine deposition, covering a wide area, was collected on the counterelectrode. It was impossible to completely eliminate this electrospray emission. However, its contribution to the total mass emitted per oscillation was negligible. The reason supporting that claim is twofold. First, for an electrospray of a very conductive liquid in the cone-jet mode, the characteristic flow rate is given by [47] $Q_{e} \sim \gamma \epsilon_{0} \epsilon /(\rho K)$, so one would expect that the flow rate carried by the electrospray was of that order. Comparing the former with the total flow rate, one obtains

$$
\frac{Q_{e}}{Q} \sim \frac{\gamma \epsilon_{0} \epsilon}{\rho K Q}=\frac{t_{e} / t_{c}}{q} \ll 1 .
$$

Some preliminary experiments varying the liquid conductivity seem to support Eq. (5) in the sense that the ratio $Q_{e} / Q$ decreases as $K$ increases, although an investigation of its precise dependence on the conductivity is beyond the scope of the present work. In principle, Eq. (5) also provides a minimum flow rate criterion, namely that $Q>Q_{e}$, since no main droplet will be emitted if the entire flow rate is emitted as an electrospray. However, experiments performed with very small flow rates show that the periodic microdripping regime gives way to a pulsed nonperiodic emission akin to axial spray mode I of Juraschek and Röllgen [29] well before values of the order of $Q_{e}$ are reached.

Second, in all the experiments we compared the value of the diameter of the emitted droplet measured from the images with the one obtained from the mass conservation balance (including the measured oscillation frequency). The differences were within the measurement errors. In conclusion, the mass emitted in the form of an ultrafine electrospray is negligible, so we shall consider that the total mass is emitted in the form of a single drop. From a practical point of view, even though electrospray emission might be an undesirable effect of this microdripping regime, the fact that these ultrafine droplets are highly charged (high electrical mobility) allows sweeping them out very easily by using nearby electrodes, while the main droplet may fly away carried by its own inertia.

\section{Ligament characterization}

Figure 13 shows the dimensionless length and width of the ligament at the tip of the meniscus immediately before a droplet is ejected $\left(\ell_{s}=L_{s} / D\right.$ and $\left.d_{s}=D_{s} / D\right)$ as functions of the dimensionless flow rate for three values of $B_{E}$. These results suggest that $\ell_{s}$ and $d_{s}$ increase as powers of $q$, although these powers may be different for the very small values of $q$
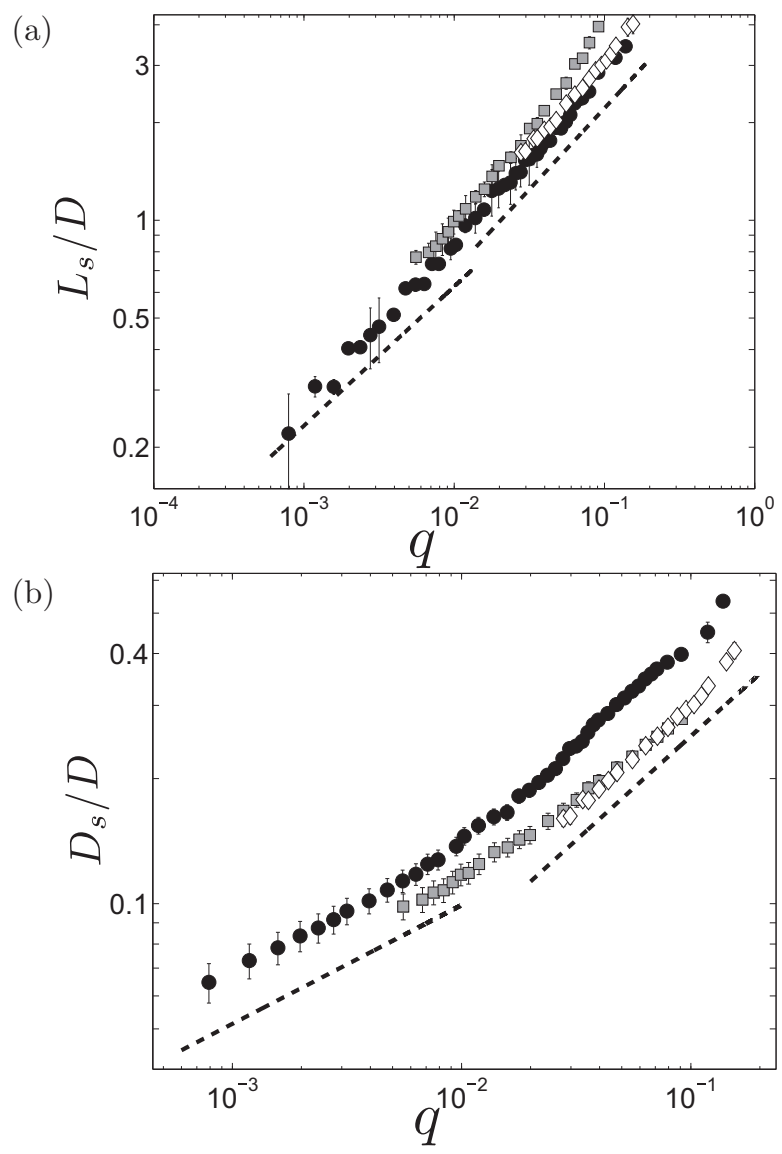

FIG. 13. Dimensionless length and width of the ligament immediately before detachment as functions of the dimensionless flow rate for $B_{E}=0.20$ (circles), 0.27 (squares), and 0.37 (diamonds). The dashed straight lines at the left-hand sides have slopes (a) 3/7 (for $L_{s} / D$ ) and (b) $2 / 7$ (for $D_{s} / D$ ). The dashed lines at the right-hand side have slope $1 / 2$.

corresponding to the plateaus of Figs. 7 and 8 and for larger values of this parameter.

The conditions around the tip of the meniscus when the ligament develops are too complex to allow a simple analysis of the process, which seems to depend on the competition of electric stresses trying to elongate the ligament and the retraction of the rest of the tip, that probably pushes the liquid toward the bulk of the meniscus. At least for very small values of $q$, the process appears to be local, in the sense that it does not affect the evolution of the rest of the meniscus, and short compared to the period of the oscillation. Tentative estimations for the latest stages of the ligament evolution are worked out in this section.

The results for the smallest values of $q$ in Fig. 13 can be partially rationalized as follows. As mentioned in Sec. III B, inspection of a number of video records suggests that a ligament appears in these cases because, after reaching its maximum elongation, most of the meniscus begins receding under the effect of surface tension stresses while locally intense electric stresses still keep its tip pinned. Assuming that recession occurs at the capillary velocity $v_{c}$ (from the balance of inertia and surface tension in the bulk of the meniscus) and that the time available for the ligament to 
form is of the order of the pinchoff time $t_{s}=\left(\rho D_{s}^{3} / \gamma\right)^{1 / 2}$ determined from the balance of inertia and surface tension in the ligament $\left(\rho D_{s}^{2} / t_{s}^{2} \sim \gamma / D_{s}\right)$, the length of the ligament would be $L_{s} \sim v_{c} t_{s}=D_{s}^{3 / 2} / D^{1 / 2}$. In addition, the volume of the detaching ligament must coincide with the volume of liquid supplied to the meniscus during a period of the oscillation, which is of the order of the capillary time, i.e., $D_{s}^{2} L_{s} \sim Q t_{c}$. These two conditions taken together give $L_{s} / D \sim q^{3 / 7}$ and $D_{s} / D \sim q^{2 / 7}$, which are represented by the dashed lines on the left-hand side of Figs. 13(a) and 13(b), respectively. However, the consistency of these estimations is not immediately clear. At first sight, since the only dimensionless control parameters of the problem are $q$ and $B_{E}$, the proportionality factors implicit in the proposed power laws can be at most functions of $B_{E}$ and should be expected to be of order unity because $B_{E}=$ $O$ (1). This would imply that $L_{s} \ll D_{s}$ for any $q \ll 1$, which is meaningless. The measured aspect ratio of the detaching ligament is $L_{s} / D_{s}=4-8$ in our experiments, indicating that the proportionality factor for $D_{s} / D$ is small compared with that for $L_{s} / D$ in the small region of the parameter space where this type of microdripping occurs.

The evolution of the meniscus undergoes some changes when the flow rate $q$ increases. First, the period of the oscillation increases with $q$ past the plateau on the left-hand side of Fig. 8. Redoing the estimations above with a period proportional to $q^{1 / 2}$, the modified results $L_{s} / D \sim q^{9 / 14}$ and $D_{s} / D \sim q^{3 / 7}$ are obtained, whose larger exponents are in qualitative agreement with the increasing slopes on the righthand side of Figs. 13(a) and 13(b). Second, the elongation of the meniscus increases with $q$, which modifies the process of formation of a ligament. The process changes from the axial growth due to differential receding velocities discussed above to a radial squeezing of a region of the elongated meniscus around its tip; compare Figs. 5(a) and 5(b). The length of this region eventually becomes of the order of the total length of the meniscus, though the volume emitted per cycle is still small compared to the volume of the meniscus because the ligament undergoes substantial radial squeezing before detachment. The elongation time of the meniscus is $T \sim$ $L_{S} / v_{E} \sim \rho^{1 / 2} L_{S} / \epsilon_{0}^{1 / 2} E$ in these conditions, where the velocity induced in the liquid by electric stresses (from the balance $\rho v_{E}^{2} \sim \epsilon_{0} E^{2}$ ) has been used. This estimation is equivalent to that of Sec. III A with $L_{s}$ playing the role of the length of the meniscus. Assuming that the period of the oscillation is of the order of the elongation time, the volume conservation condition $D_{s}^{2} L_{s} \sim Q T$ gives $D_{s} / D \sim q^{1 / 2} / B_{E}^{1 / 4}$, while the value of $L_{s}$ is not determined by these estimations. The square root increase of the width of the ligament with dimensionless flow rate agrees with the results of Fig. 13(b) (dashed line on the right-hand side of this figure).

Figure 13(a) shows that the length of the ligament also increases nearly as the square root of $q$. This result can be understood by noticing that (i) electric stresses are needed to partially offset surface tension stresses in the prominent, elongated ligament that develops for large values of $q$ [see, e.g., Fig. 5(b)], which requires $\epsilon_{0} E_{n}^{2} \sim \gamma / D_{s}$, where $E_{n}$ is the electric field at the surface of the ligament, and (ii) the measured electric charge of the detached droplets is of the order of the limit Rayleigh charge $\mathcal{C}_{R}=\left(8 \pi^{2} \epsilon_{0} \gamma D_{d}^{3}\right)^{1 / 2}$.
The ratio of the measured charge to the limit charge is about $1 / 4$ and depends little on $q$ and $B_{E}$; see Fig. 10. The conditions that the charge and volume of a droplet should coincide with the charge and volume of the ligament immediately before detachment read $\sigma D_{s} L_{s} \sim \mathcal{C}_{R}$ and $D_{s}^{2} L_{s} \sim D_{d}^{3}$, where $\sigma=\epsilon_{0} E_{n}$ is the surface density of charge in the ligament [49]. Upon eliminating $D_{d}$ and $E_{n}$, these conditions give $L_{s} \sim D_{s}$ up to a numerical factor that turns out to be on the order of 6-7.

\section{E. High- $q$ trends and droplet diameter}

The estimation $L_{s} / D \sim q^{1 / 2} / B_{E}^{1 / 4}$ (up to a numerical factor) accounts for the increase of the dimensionless volume of the meniscus on the right-hand side of Fig. 7. Also, together with the estimation of the elongation time worked out in Sec. III D, which can be recast as $f \sim B_{E}^{1 / 2} /\left(L_{S} / D\right)$ in dimensionless variables, it accounts for the decrease of the dimensionless frequency on the right-hand side of Fig. 8.

The dimensionless equivalent droplet diameter in Fig. 9 increases as $q^{1 / 3}$ for very small flow rates and as $q^{1 / 2}$ for larger flow rates. Both results follow from the volume conservation condition $d_{d}=(6 / \pi)^{1 / 3}(q / f)^{1 / 3}$ noticing that $f=O(1)$ for small $q$ and $f=O\left(q^{-1 / 2}\right)$ for larger $q$. It is noteworthy that the type of microdripping analyzed here can be operated to yield monodisperse droplets whose diameter is one-tenth of the diameter of the capillary tube to which the oscillating meniscus is attached.

\section{F. Comparisons with other results in the literature}

For comparison, some experimental data of Juraschek and Röllgen [29] for the meniscus pulsation frequency as a function of $q$ and $B_{E}$ have been included in Figs. 8 and 11 (open triangles). Since these results have been used for reference in much of the later work (see, e.g., Refs. [31,32]), the good agreement displayed by this comparison shows that our results fit into the body of known data. Some of the pictures of Juraschek and Röllgen [29] suggest that the contact line of their menisci with the capillary tube may in some cases spread slightly along the outer surface of the tube. While the liquid wetting this surface is not expected to play an important part in the oscillation of the meniscus, because its motion is hindered by the proximity of the wall, it still enlarges the radius of the liquid surface and thus decreases the electric field acting on it. To try to account for this effect, an arbitrary reduction factor has been included in the expression of the electric field $E$ in (4), whose value has been chosen for the plateaus in Fig. 13 of Juraschek and Röllgen and in our Fig. 8 to coincide. The good agreement achieved when this same factor is used for the data in Fig. 11 partially justifies its use.

A number of scaling laws for the meniscus oscillation frequency in pulsating spray modes have been proposed by different authors. Marginean et al. [30] note that the oscillation frequency of low-conductivity liquids in large emitters is remarkably close to the lowest capillary frequency of an isolated spherical droplet of radius equal to the radius of the contact line, which is given by $f=4 / \pi$ in our dimensionless variables and is marked by the solid line in Fig. 8. Following Jurascheck and Röllgen [29], these authors attribute the increase of the oscillation frequency with the voltage applied 
to the meniscus to a decrease of the amount of liquid pulsating at the end of the capillary. Similarly, they explain the decline of the frequency with increasing flow rate as an effect of the increase of liquid volume. Using the estimate of Marginean et al. as a starting point, Choi et al. [32] work out the modified scaling $f \sim B_{E}^{3 / 4}$ by replacing the radius of the contact line by the radius of the ejected ligament, which they estimate independently. Their result is included in Fig. 11 (dashed line). It qualitatively accounts for the trend of the data, although it does not include the effect of the flow rate on the frequency.

Some numerical results for axial spray mode II have been reported in Ref. [37] for an inviscid liquid of infinite electrical conductivity that is injected at a constant flow rate through an orifice in a metallic plate into a region of uniform electric field. The numerical results reproduce the main features of the periodic dynamics, including the stretching of the meniscus, the formation of a ligament whose tip emits a spray of tiny droplets and eventually detaches, and the subsequent recoil of the meniscus. However, the simplified configuration used in the numerical simulations precludes quantitative comparison with our experimental data. Thus, for a value of the dimensionless flow rate in the range of our experiments, the numerical values of the electric Bond number required for periodic microdripping (based on the uniform electric field far from the meniscus) are somewhat larger than the experimental values. The computed oscillation frequency and the maximum length of the ligament are somewhat larger than in the experiments and the mean volume of the meniscus is significantly smaller. These differences can be traced to the morphology of the electric field. Contrary to the case of a meniscus at the end of a capillary, the electric field does not tend to zero far from a meniscus on a metallic plate. This leads to enhanced electric stresses that accelerate the formation of a ligament, increase its elongation, and cause its detachment before the volume of the meniscus can increase much. In addition, since the flow rate is given, the faster dynamics also implies a larger dripping frequency.

\section{CONCLUSION}

We have studied the electric microdripping regime of highly conducting, low-viscosity liquids as a means to generate monodisperse droplets with diameter considerably smaller than the capillary tube holding the dripping meniscus. We have focused on the conditions in which the microdripping is periodic.
During each oscillation, the meniscus elongates axially, growing an axisymmetric disturbance that eventually leads to the formation of a ligament and a droplet. Simultaneously, the meniscus develops a pointed tip that emits an ultrafine aerosol for a non-negligible fraction of the cycle. However, in the conditions investigated, almost all the mass emitted per oscillation is contained in the main droplet. The ultrafine emission seems to evolve on its own, independently of the main breakup process.

The dimensionless parameters controlling this periodic microdripping regime are the electric Bond number $B_{E}$ and the dimensionless liquid flow rate $q$. The microdripping regime occurs in a fairly narrow range of values of $B_{E}$ of order unity. The mean volume of the meniscus in an oscillation cycle is of the order of the cube of the diameter of the capillary tube for very small values of the flow rate and increases nearly as the square root of the flow rate when this parameter increases. The oscillation frequency nondimensionalized with the capillary time is of order unity for very small $q$ and decreases as $1 / q^{1 / 2}$ when $q$ increases. Approximate scaling laws valid in different ranges of $q$ have been worked out for the length and width of the ligament and for the diameter of the main droplets.

By reducing the liquid flow rate, this mode of periodic microdripping can easily yield monodisperse droplets with diameters one-tenth of that of the capillary tube at rates of the order of the capillary frequency. In the data shown, monodisperse droplets of $50 \mu \mathrm{m}$ from a nozzle of $500 \mu \mathrm{m}$ are produced at rates of several $\mathrm{KHz}$, thus demonstrating that this microdripping regime constitutes a real alternative to the hydrodynamic atomization techniques currently used for printing or for material processing. The scaling laws obtained should allow designing the geometry of the nozzle device together with the properties of the liquid to set a given droplet size range, potentially including submicronic, within which monodisperse droplets may be produced at will.

\section{ACKNOWLEDGMENTS}

This work was supported by the Spanish Ministerio de Economía y Competitividad through Projects No. DPI201020450-C03 and No. DPI2013-47372-C02. S.E.I. and F.J.H. also acknowledge support through Project No. CSD201000010. Very fruitful discussions with Professor FernándezNieves, from Georgia Tech, are acknowledged. We also thank I. Sánchez-Escudero for her assistance with laboratory work.
[1] M. Cloupeau and B. Prunet-Foch, Electrohydrodynamic spraying functioning modes: A critical review, J. Aerosol Sci. 25, 1021 (1994).

[2] A. Jaworek and A. Krupa, Classification of the modes of EHD spraying, J. Aerosol Sci. 30, 873 (1999).

[3] J. B. Fenn, M. Mann, C. K. Meng, S. F. Wong, and C. M. Whitehouse, Electrospray ionization for mass spectrometry of large biomolecules, Science 246, 64 (1989).

[4] J. Fernández de la Mora, The fluid dynamics of Taylor cones, Annu. Rev. Fluid Mech. 39, 217 (2007).
[5] Y. Wang, M. K. Tan, D. B. Go, and H. C. Chang, Electrospray cone-jet breakup and droplet production for electrolyte solutions, Europhys. Lett. 99, 64003 (2012).

[6] C. Larriba and J. Fernández de la Mora, Production of monodisperse submicron drops of dielectric liquids by charge-injection from highly conducting liquids, Phys. Fluids 23, 102003 (2011).

[7] P. Miao, W. Balachandran, and P. Xiao, Formation of ceramic thin films using electrospray in cone-jet mode, IEEE Trans. Ind. Appl. 38, 50 (2002). 
[8] O. Basaran, H. Gao, and P. Bhat, Nonstandard inkjets, Annu Rev. Fluid Mech. 45, 85 (2013).

[9] A. Schober, R. Gunther, A. Schwienhorst, M. Doring, and B. F. Lindemann, Accurate high-speed liquid handling of very small biological samples, BioTechniques 15, 324 (1993).

[10] T. C. Tisone, Dispensing systems for miniaturized diagnostics, IVD Technol. SANTA MONICA, CA, US 4, 40 (1998).

[11] E. Litborn, M. Stjernström, and J. Roeraade, Nanoliter titration based on piezoelectric drop on demand technology and laserinduced fluorescence detection, Anal. Chem. 70, 4847 (1998).

[12] G. Allmaier, Picoliter to nanoliter deposition of peptide and protein solutions for matrix assisted laser desorption/ionization mass spectrometry, Rapid Commun. Mass Spectrom. 11, 1567 (1997).

[13] J. Kling, MALDI chip shot, Anal. Chem. 73, 68A (2001).

[14] H. Hulett, W. Bonner, J. Barrett, and L. Herzenberg, Cell sorting: Automated separation of mammalian cells as a function of intracellular fluorescence, Science 166, 747 (1969).

[15] M. Chee, R. Yang, E. Hubbell, A. Berno, X. C. Huang, D. Stern, J. Winkler, D. J. Lockhart, M. S. Morris, and S. P. A. Fodor, Accessing genetic information with high-density DNA arrays, Science 274, 610 (1996).

[16] T. P. Theriault, S. C. Winder, and R. C. Gamble, Application of ink-jet printing technology to the manufacture of molecular arrays, in DNA Microarrays: A Practical Approach, edited by M. Schena (Oxford University Press, Oxford, 1999), pp. 101119.

[17] D. Englert, Production of microarrays on porous substrates using noncontacting piezoelectric dispensing, in Microarray Biochip Technology, edited by M. Schena (Eaton, Natick, 2000), pp. 231-246.

[18] W. R. Cox, T. Chen, C. Guan, D. J. Hayes, R. E. Hoenigman, B. T. Teipen, and D. L. MacFarlane, Micro-jet printing of refractive microlenses, in Optical Society of America. Diffractive Optics and Micro-Optics. Summer Topical Meetings, Kailua-Kona, Hawaii, 1998, OSA Technical Digest Series Vol. 10 (Optical Society of America, Washington DC, 1998), pp. 276-278.

[19] W. R. Cox, C. Guan, D. J. Hayes, and D. B. Wallace, Microjet printing of micro-optical interconnects, Int. J. Microcircuits Electron. Packag. 23, 346 (2000).

[20] F. Q. Gao and A. A. Sonin, Precise deposition of molten microdrops: The physics of digital microfabrication, Proc. R. Soc. London Ser. A 444, 533 (1994).

[21] S. Ashley, Rapid prototyping is coming of age, Mech. Eng. 117, 62 (1995).

[22] D. B. Wallace, Automated electronic circuit manufacturing using ink-jet technology, Trans. ASME J. Electron. Packag. 111, 108 (1989).

[23] D. J. Hayes, W. R. Cox, and M. E. Grove, Low-cost display assembly and interconnects usingink-jet printing technology, $\mathrm{J}$. Soc. Inf. Display 9, 9 (2001).

[24] D. J. Hayes, D. B. Wallace, and W. R. Cox, MicroJet printing of solder and polymers for multi-chip modules and chip-scale packages, Proceedings of SPIE - The International Society for Optical Engineering 3830, 242 (1999).

[25] M. Orme, J. Courter, Q. Liu, C. Huang, and R. Smith, Electrostatic charging and detection of nonconventional droplet streams formed from capillary stream breakup, Phys. Fluids 12, 2224 (2000).
[26] R. N. Udey, A. D. Jones, and G. R. Farquar, Aerosol and microparticle generation using a commercial inkjet printer, Aerosol Sci. Technol. 47, 361 (2013).

[27] V. van Steijn, P. M. Korczyk, L. Derzsi, A. R. Abate, D. A. Weitz, and P. Garstecki, Block-and-break generation of microdroplets with fixed volume, Biomicrofluidics 7, 024108 (2013).

[28] A. Patrascioiu, M. Duocastella, J. M. Fernndez-Pradas, J. L. Morenza, and P. Serra, Liquids microprinting through a novel film-free femtosecond laser based technique, Appl. Surf. Sci. 257, 5190 (2011).

[29] R. Juraschek and F. W. Röllgen, Pulsation phenomena during electrospray ionization, Int. J. Mass Spectrom. 177, 1 (1998).

[30] I. Marginean, P. Nemes, L. Parvin, and A. Vertes, How much charge is there on a pulsating Taylor cone?, Appl. Phys. Lett. 89, 064104 (2006).

[31] C. H. Chen, D. A. Saville, and I. A. Aksay, Scaling laws for pulsed electrohydrodynamic drop formation, Appl. Phys. Lett. 89, 124103 (2006).

[32] H. K. Choi, J. Park, O. Park, P. M. Ferreira, J. G. Georgiadis, and J. A. Rogers, Scaling laws for jet pulsations associated with high-resolution electrohydrodynamic printing, Appl. Phys. Lett. 92, 123109 (2008).

[33] I. Marginean, P. Nemes, and A. Vertes, Astable regime in electrosprays, Phys. Rev. E 76, 026320 (2007).

[34] J. Kim, H. Oh, and S. S. Kim, Electrohydrodynamic dropon-demand patterning in pulsed cone-jet mode at various frequencies, J. Aerosol Sci. 39, 819 (2008).

[35] D. K. Kang, M. W. Lee, H. Y. Kim, S. C. James, and S. S. Yoon, Electrohydrodynamic pulsed-inkjet characteristics of various inks containing aluminum particles, J. Aerosol Sci. 42, 621 (2011).

[36] M. W. Lee, D. K. Kang, N. Y. Kim, H. Y. Kim, S. C. James, and S. S. Yoon, A study of ejection modes for pulsed-DC electrohydrodynamic inkjet printing, J. Aerosol Sci. 46, 1 (2012).

[37] F. J. Higuera, S. E. Ibáñez, A. J. Hijano, and I. G. Loscertales, Pulsating emission of droplets from an electrified meniscus, J. Aerosol Sci. 66, 193 (2013).

[38] S. Mishra, K. I. Barton, A. G. Alleyne, P. M. Ferreira, and J. A. Rogers, High-speed and drop-on-demand printing with a pulsed electrohydrodynamic jet, J. Micromech. Microeng. 20, 095026 (2010).

[39] E. Sutanto, K. Shigeta, Y. K. Kim, P. G. Graf, D. J. Hoelzle, K. L. Barton, P. M. Ferreira, A. G. Alleyn, and J. A. Rogers, A multimaterial electrohydrodynamic jet (E-jet) printing system, J. Micromech. Microeng. 22, 045008 (2012).

[40] P. Galliker, J. Schneider, H. Eghlidi, S. Kress, V. Sandoghdar, and D. Poulikakos, Direct printing of nanostructures by electrostatic autofocussing of ink nanodroplets, Nat. Commun. 3, 890 (2012).

[41] S. Lee, D. Byun, D. Jung, J. Choi, Y. Kim, J. H. Yang, S. U. Son, S. B. Q. Tran, and H. S. Ko, Pole-type ground electrode in nozzle for electrostatic field induced drop-on-demand inkjet head, Sensors Actuat. A 141, 506 (2008).

[42] J. Canny, A computational approach to edge detection, IEEE Trans. Pattern Anal. Mach. Intell. PAMI-8, 679 (1986).

[43] A. J. Acero, C. Ferrera, J. M. Montanero, M. A. Herrada, and J. M. López-Herrera, Experimental analysis of the evolution of an electrified drop following high voltage switching, Eur. J. Mech. B 38, 58 (2013). 
[44] B. Song and J. Springer, Determination of interfacial tension from the profile of a pendant drop using computer-aided image processing, J. Colloid Interface Sci. 184, 77 (1996).

[45] R. Clift, J. R. Grace, and M. E. Weber, Bubbles, Particles and Drops (Academic, New York, 1978).

[46] G. I. Taylor, Electrically driven jets, Proc. R. Soc. London Ser. A 313, 453 (1969).
[47] J. Fernández de la Mora and I. G. Loscertales, The current emitted by highly conducting Taylor cones, J. Fluid Mech. 260, 155 (1994).

[48] Lord Rayleigh, On the equilibrium of liquid conducting masses charged with electricity, Philos. Mag. Ser. 5 14, 184 (1882).

[49] L. D. Landau and E. M. Lifshitz, Electrodynamics of Continuous Media (Pergamon, Oxford, 1960). 